\title{
A Practical Method for Ablation Catheter Reintroduction into the Left Atrium via Prior Transseptal Puncture, without Radiation
}

\author{
Ling-pin Pang, MD, ${ }^{1,3 *}$ Song-wen Chen, MD, PhD, ${ }^{2 *}$ Gen-qing Zhou, MD, ${ }^{2}$ Yong Wei, MD, PhD, \\ Can Chen, MD, PhD, ${ }^{1,3}$ Shi-an Huang, MD ${ }^{3}$ Shao-wen Liu, MD, PhD ${ }^{2}$ \\ ${ }^{1}$ The First Affiliated Hospital, Jinan University, Guangzhou, Guangdong, China; ${ }^{2}$ Department of Cardiology, Shanghai \\ General Hospital, Shanghai Jiao Tong University School of Medicine, Shanghai, China; ${ }^{3}$ Department of Cardiology, Affiliated \\ Hospital of Guangdong Medical University, Zhanjiang, Guangdong, China
}

\section{ABSTRACT}

Background: We evaluated the feasibility and safety of reintroducing an ablation catheter (ABL) into the left atrium (LA) through a previously punctured interatrial septum under guidance of the show-catheter image-track function of the CARTO 3 3-dimensional (3D) electroanatomic mapping system.

Methods: One hundred consecutive paroxysmal or persistent drug-refractory atrial fibrillation (AF) patients (men: 55 ; mean age, $64.7 \pm 12.1$ years) who had undergone 2 fluoroscopy-guided transseptal punctures and anatomical LA reconstruction under CARTO 3-guidance, and required ABL reinsertion into the LA during mapping or ablation, were included. They were randomized 1:1 to the show-catheter (reintroduction under the CARTO 3 show-catheter imagetrack function) or fluoroscopy group (reintroduction under conventional fluoroscopy).

Results: Although the reconstructed 3D anatomy map was displaced in $21 / 100$ patients (21.0\%), the ABL was successfully reintroduced in all patients. In the show-catheter and fluoroscopy groups, model displacement incidence (18\% versus $24 \%$ ), tachyarrhythmias ( $46.0 \%$ versus $52.0 \%$ ), complications ( $2 \%$ versus $4 \%$ ), and number of ABLs reintroduced into the LA $(3.3 \pm 0.8$ versus $3.1 \pm 0.9)$ were similar (all $P>$ $.05)$. The show-catheter group displayed shorter ABL reintroduction time $(9.5 \pm 5.5 \mathrm{~s}$ versus $156.4 \pm 35.5 \mathrm{~s}, P<.01)$, $\mathrm{ABL}$ reintroduction $\mathrm{X}$-ray exposure time $(0 \mathrm{~s}$ versus $39.3 \pm$

Received fune 21, 2019; received in revised form September 3, 2019; accepted September 5, 2019.

*These authors contributed equally to this work.

This study was supported by the Shenkang Hospital Development Centre of Shanghai (No. 16CR1015A), the Science and Technology Planning Project of Zhanjiang City, China (No. 2017B01124), and the Scientific Research Fund of Guangdong Medical University (No. M2017004).

Correspondence: Ling-pin Pang, MD, Department of Cardiology, Affiliated Hospital of Guangdong Medical University, Zhanjiang, Guangdong, China; +86 18319120186; (e-mail: panglingpin@126.com); Can Chen, PhD, Department of Cardiology, Affiliated Hospital of Guangdong Medical University, Zhanjiang, Guangdong, China. +86 13902502296; (e-mail: chencan-21@163.com).
$13.8 \mathrm{~s}, P<.01)$, and total $\mathrm{X}$-ray exposure time $(4.1 \pm 1.4 \mathrm{~min}$ versus $4.7 \pm 0.8, P<.05)$.

Conclusion: During AF ablation, the catheter can be safely reintroduced into the LA, without additional fluoroscopy, under guidance of the CARTO 3 show-catheter image track function.

\section{INTRODUCTION}

The use of three-dimensional (3D) mapping systems has greatly improved radiofrequency catheter ablation (RFCA) for arrhythmias by providing mapping of cardiac structure and electrocardiography signals, while markedly shortening procedure duration and reducing dependence on X-ray imaging [Christoph 2015]. However, gaining access to the left side of the heart requires transseptal puncture (TSP), typically under fluoroscopic guidance [Earley 2009]. Moreover, intentionally or unintentionally switching the ablation catheter (ABL) between the atria 2-4 times in cases with paroxysmal or persistent atrial fibrillation (AF) [Alhajiri 2014; Morady 2009] has been associated with an increased risk of complications, increased X-ray exposure, and a longer procedure duration [Gul 2017; Klimek-Piotrowska 2016b; Meisinger 2016]. Although intracardiac ultrasound (ICE) for TSP has become routine at some institutions, its use remains limited due to its prohibitive cost.

The present randomized study compared the effectiveness of AF ablation with ABL switching between atria through the interatrial septum under the guidance of either the fluoroscopy-free show-catheter image track function of the CARTO 3 mapping system or fluoroscopy.

\section{MATERIALS AND METHODS}

\section{Study Population}

From January 2017 to October 2018, a total of 100 consecutive patients (50 men; mean age, $64.7 \pm 12.1$ years) with paroxysmal or persistent $\mathrm{AF}$ underwent their first ablation procedure at the Shanghai General Hospital, Shanghai Jiao Tong University. Only those patients who needed reintroduction of the ablation catheter into the left atrium were 


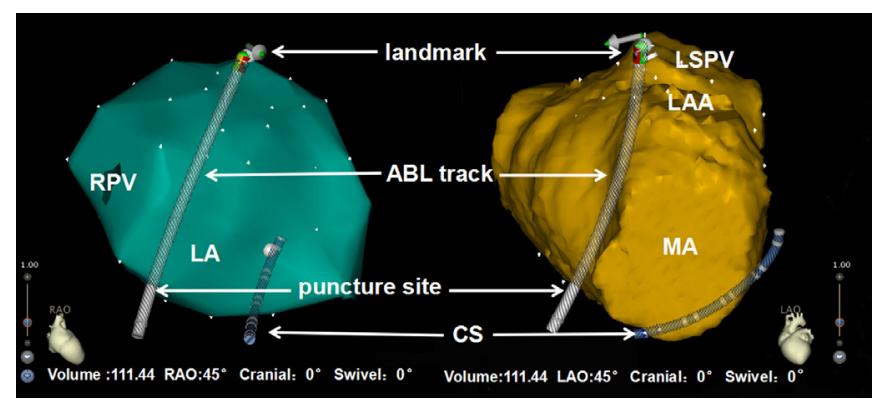

Figure 1. Creation of the catheter image-track using the show-catheter function. The ablation catheter image-track was created under guidance of the show-catheter image track function of the CARTO 3 mapping system. The $45^{\circ}$ right anterior oblique and left anterior oblique $45^{\circ}$ views were shown. After successful reconstruction of the left atrial anatomy, the SL1 Swartz sheath was withdrawn into the right atrium to show the ablation catheter tip and the proximal catheter. The ablation catheter was manipulated carefully to make stable contact with the anterior wall of the left atrium. With the proximal catheter across the interatrial septum, a white landmark was acquired and created to show the location of the septal puncture. $A B L$ indicates ablation catheter; $L A$, left atrium; CS, coronary sinus; MA, mitral annulus; LPV, left pulmonary vein; RPV, right pulmonary vein; LAA, left atrial appendage.

prospectively enrolled in this study. The institutional ethics committee approved the study protocol, and all patients provided written informed consent.

\section{Preoperative Preparation}

The preoperative preparation and ablation protocol have previously been described in detail [Chen 2012; Chen 2019]. Ablation was performed under sedation with midazolam and fentanyl [Chen 2019]. All patients with more than one thromboembolism risk factor (CHADS2 score $\geq 1$ ) received oral anticoagulation with rivaroxaban, dabigatran, or warfarin, based on an international normalized ratio in the range of 2.0-3.0, at least 3 weeks before ablation. On the day of ablation, patients did not take anticoagulants. Routine examinations, such as transthoracic echocardiography, transesophageal echocardiography, and pulmonary vein (PV) computed tomography angiography, were performed within 3 days before ablation to exclude contraindications, particularly thrombi within the heart chambers.

\section{AF Ablation Procedure}

Patients first underwent 2 TSPs guided by fluoroscopy. After transseptal catheterization, an intravenous bolus of heparin (100 $\mathrm{U} / \mathrm{kg}$ ) was administered, activated clotting time was monitored every 15 minutes, and continuous heparin infusion was administered to control activated clotting time within 250-350 s.

Nonselective PV angiography was performed to identify PV ostia. Contact force catheters (SmartTouch, Biosense Webster, Irvine, CA, USA) were used for mapping and ablation in 100 patients.

After anatomical image reconstruction of the left atrium (LA), computed tomography images were integrated by reconstruction under the guidance of a CARTOMerge (Biosense Webster), with a single landmark in the

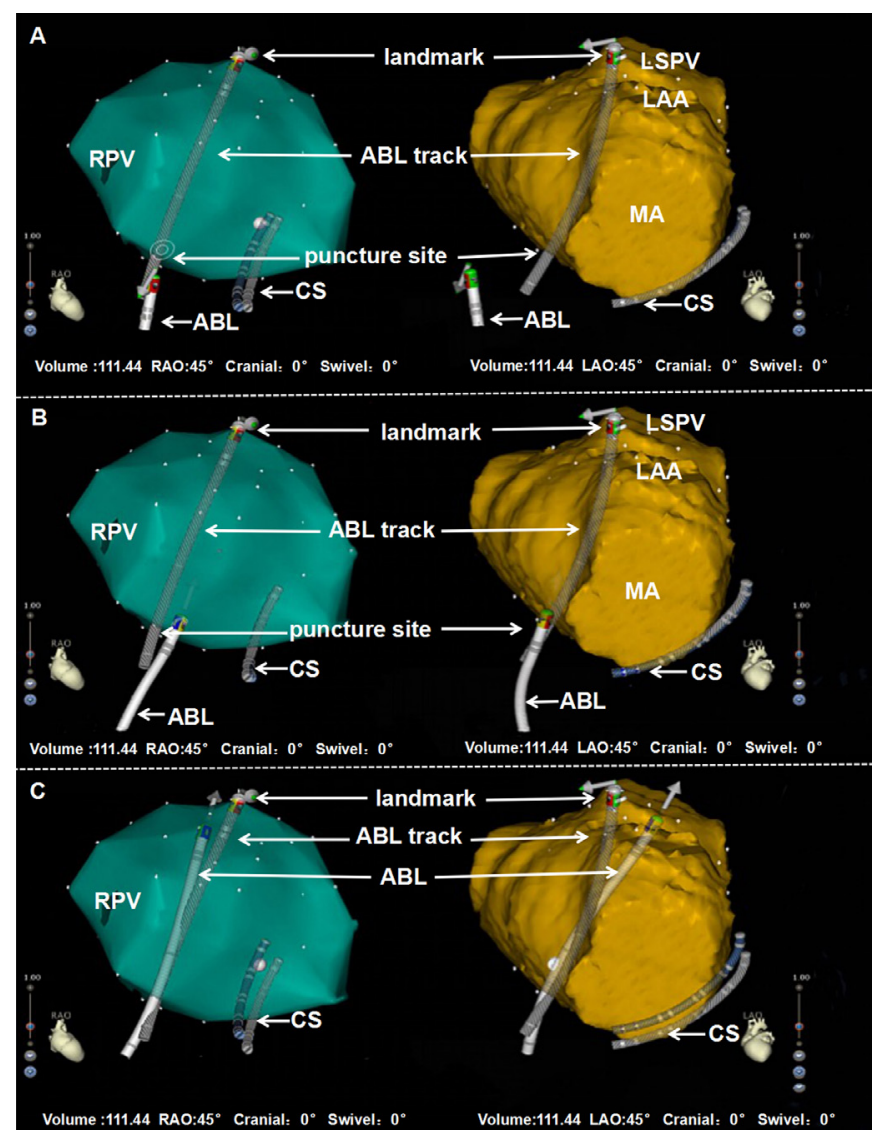

Figure 2. Manipulation for reintroducing the ablation catheter into the left atrium under the guidance of the show-catheter image-tract. A. The ablation catheter was introduced into the interatrial septum as close as possible to the targeted region. B. Under the guidance of the showcatheter image-tract, the direction of the ablation catheter was adjusted to find the accurate location of the septal puncture. $C$. The ablation catheter was reintroduced into the left atrium via the prior punctured septum, without requiring fluoroscopy. 3D indicates three-dimensional; $A B L$, ablation catheter; AF, atrial fibrillation; FAM, fast anatomic mapping; ICE, intracardiac ultrasound; LA, left atrium; PV, pulmonary vein; RFCA, radiofrequency catheter ablation; TSP, transseptal puncture.

posterior-inferior wall of the right inferior PV. Another landmark, at the anterior-superior wall of the LA, was acquired with the ablation catheter crossing the septum (Figure 1).

This was achieved using the following steps:

Step 1 . The ablation catheter was manipulated carefully to make stable contact with the anterior wall of the LA using $45^{\circ}$ right anterior oblique (RAO) and left anterior oblique (LAO) views.

Step 2. The SL1 Swartz sheath was withdrawn into the right atrium to reveal the ablation catheter tip. The proximal catheter, crossing the interatrial septum, was also clearly displayed in the CARTO 3 system (Biosense Webster).

Step 3. A white landmark was acquired and created. Using the show-catheter function in CARTO 3, the image track of the ABL and the location of the punctured septum were displayed with this landmark, which was retained. 
Table 1. Comparison between the Show-Catheter Group and the Fluoroscopy Group

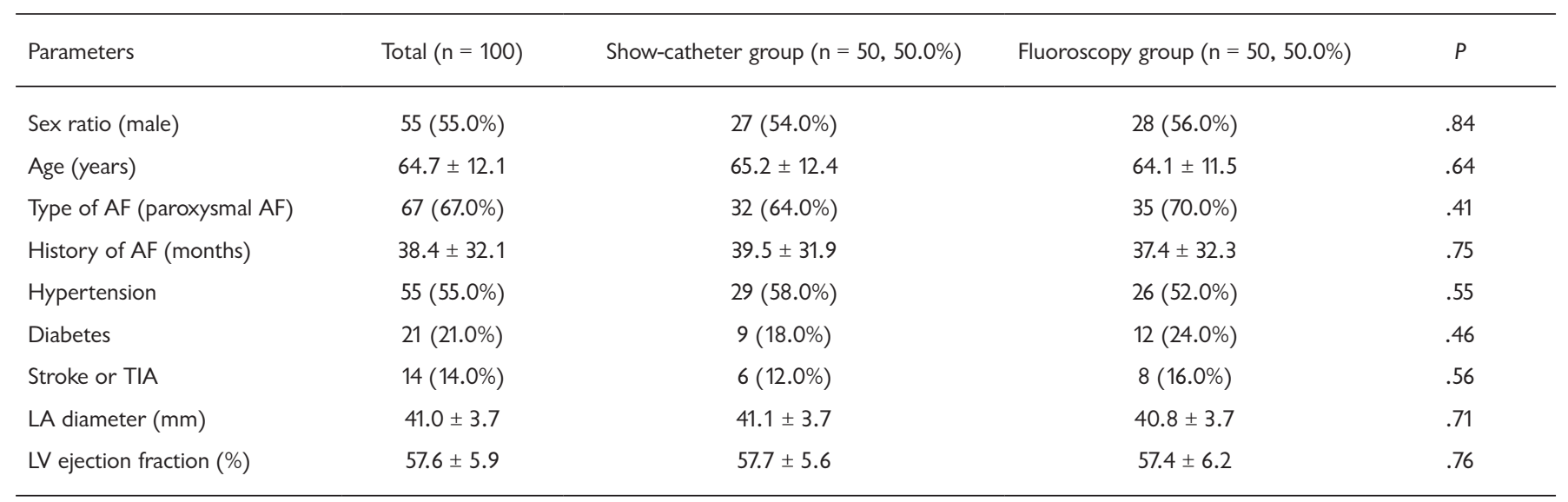

AF indicates atrial fibrillation; TIA, transient ischemic attack; LA, left atrial; LV, left ventricular.

Circumferential PV isolation was performed immediately outside the ostia of the ipsilateral PVs under the guidance of a CARTO-Merge and a circular mapping catheter (Lasso, Biosense Webster). The ipsilateral PVs were isolated as pairs, without ablation, through the carina in all patients.

Patients with atrial arrhythmias after $\mathrm{PV}$ isolation underwent linear ablation (including the LA roof, mitral isthmus, and cavotricuspid isthmus, among others) and complex fractionated atrial electrogram ablations, if necessary. Entrainment mapping and activation mapping were performed to identify the mechanisms of regular atrial arrhythmias, such as atrial flutter and atrial tachycardia, during the procedure.

\section{Reintroduction of the ABL into the $L A$}

Patients requiring RA/LA transfers for mapping or ablation (i.e. repeated withdrawal of the ABL from the LA into the RA, followed by reentry to the LA from the RA) were randomized in a 1:1 ratio to the show-catheter group (ABL was reintroduced into the LA under the guidance of the show-catheter image-track function of the CARTO 3 system) or to the fluoroscopy group (ABL was reintroduced into the LA under conventional fluoroscopy guidance). The ABL was reintroduced into the LA from the RA as many times as needed in both groups.

\section{Show-Catheter Group}

In the show-catheter group, the $\mathrm{ABL}$ was reintroduced into the LA as follows (Figure 2):

Step 1. Using the show-catheter function in CARTO 3, the white landmark was clicked, and the image track of the $\mathrm{ABL}$ and puncture site were displayed.

Step 2. Under the guidance of the show-catheter image tract, the $\mathrm{ABL}$ was introduced into the interatrial septum, as close as possible to the targeted region, using $45^{\circ} \mathrm{RAO}$ and LAO views.

Step 3. The direction of the ABL was gently adjusted to find the accurate location of the septal puncture under the guidance of the show-catheter image tract and was then reintroduced into the LA.

Step 4. Successful reintroduction of the ABL into the LA was confirmed when the head of the ABL touched the LA posterior wall.

Under the guidance of the ABL image track, the ABL was reintroduced as many times as needed into the LA from RA. If the reintroduction took more than $10 \mathrm{~min}$, TSP was repeated.

\section{Fluoroscopy Group}

Under the guidance of traditional fluoroscopy and considering the approximate position of the atrial septum in the 3D LA model, the head of the ABL was moved to the septal puncture and gently reintroduced into the LA using $45^{\circ} \mathrm{LAO}$ and $\mathrm{RAO}$ views. If the reintroduction $\mathrm{X}$-ray exposure time exceeded $10 \mathrm{~min}$, TSP was repeated.

Irrigated radiofrequency energy was delivered with an upper-temperature limit of $43^{\circ} \mathrm{C}$, a maximum radiofrequency power of $38 \mathrm{~W}$, and an infusion rate of $17-25 \mathrm{~mL} / \mathrm{min}$. In all patients, a maximal power of $25 \mathrm{~W}$ was delivered to the superior vena cava or the coronary sinus to avoid cardiac tamponade or phrenic nerve impairment.

The endpoints of persistent or long-lasting AF ablation were the termination of $\mathrm{AF}$ and conversion to sinus rhythm, including PV isolation. For paroxysmal AF, the endpoints included PV isolation and non-inducible atrial arrhythmias lasting for 5 minutes. All patients received anticoagulation for at least 3 months after ablation.

\section{Statistical Analysis}

Continuous variables were expressed as mean and standard deviation or geometric mean and interquartile range. Discrete variables were presented as percentages. Means were compared using the Student $t$ test or ANOVA. Proportions were compared by Pearson chi-square test or Fisher exact test. All tests were two-tailed. A $P$ value of $<.05$ was considered statistically significant. Statistical analyses were performed using the SPSS 17.0 software package (SPSS, Chicago, IL, USA). 
Table 2. Patient Procedure Data for Reintroduction of the Ablation Catheter

\begin{tabular}{|c|c|c|c|c|}
\hline TSP time, $\min$ & $17.2 \pm 3.6$ & $17.0 \pm 3.7$ & $17.3 \pm 3.6$ & .68 \\
\hline Number of reintroductions into $L A, n$ & $3.2 \pm 0.8$ & $3.3 \pm 0.8$ & $3.1 \pm 0.9$ & .15 \\
\hline Reintroducing time, $s$ & $83.0 \pm 77.7$ & $9.5 \pm 5.5$ & $156.4 \pm 35.5$ & $<.001$ \\
\hline Total $\mathrm{X}$-ray exposure time, min & $4.4 \pm 1.2$ & $4.1 \pm 1.4$ & $4.7 \pm 0.8$ & .010 \\
\hline Displacement of reconstructed map, $\mathrm{n}(\%)$ & $21(21.0)$ & $9(18.0)$ & $12(24.0)$ & .46 \\
\hline Complications, n (\%) & $3(3.0)$ & $1(2.0)$ & $2(4.0)$ & .56 \\
\hline
\end{tabular}

TSP indicates transseptal puncture; LA, left atrium.

\section{RESULTS}

\section{Patient Characteristics}

The study population consisted of 100 consecutive patients with paroxysmal or persistent AF. Patient characteristics are summarized in Table 1. There were no significant differences in patient characteristics between the two groups.

\section{Procedure Data and Results}

TSP and pulmonary vein isolation were successfully performed in all patients. ABL reintroduction into the LA was also successfully performed in all 100 patients. Procedure data for ABL reintroduction into the LA from the RA, through the interatrial septum, are summarized in Table 2. However, displacement of the reconstructed $3 \mathrm{D}$ anatomical map was observed in 21 of the 100 patients $(21.0 \%)$. The incidence of 3D model displacement was similar between the two groups (Table 2).

Although the number of ABL reintroductions was similar between the two groups, the reintroducing duration, reintroducing X-ray exposure duration, and total X-ray exposure duration was significantly shorter in the show-catheter group than in the fluoroscopy group (Table 2). The incidence of complications was also similar between the two groups (Table 2), with $1(2 \%)$ inguinal hematoma in the show-catheter group, and 1 (2\%) inguinal hematomas and $1(2 \%)$ pericardial tamponade (unconfirmed as procedure-related) in the fluoroscopy group.

In addition, LA roof ablation ( $\mathrm{n}=49,49.0 \%)$, mitral isthmus ablation $(\mathrm{n}=26,26.0 \%)$, cavotricuspid isthmus ablation $(\mathrm{n}=42,42.0 \%)$, and superior vena cava isolation $(\mathrm{n}=21$, $21.0 \%)$ were performed in all 100 patients, and $21(21.0 \%)$ patients required coronary sinus reinforcement ablation to obtain bidirectional conduction blockage across the MI. Linear ablation data are summarized in Table 3.

\section{DISCUSSION}

In this randomized study, we compared two imaging approaches to guide repeated switching of the ABL between the atria through the atrial septum during RFCA for AF. We found that the show-catheter image-track function of the CARTO 3 electroanatomic mapping system yielded a clearly visible catheter track. The two approaches yielded similar rates of procedural success and complication rates, but the show-catheter approach allowed easier catheter manipulation, significantly shorter procedure duration, and no X-ray exposure, as compared with fluoroscopy guidance.

In addition, the principles of the CARTO 3 electroanatomic mapping system require that the patient's body is maintained in a fixed position throughout the operation. If the patient's body is slightly moved, the computer system will sound an alarm, suggesting that the established LA image model and the actual LA position exhibit slight displacement. In our study, there was no significant difference in the incidence of displacement between the two groups; thus, there was no significant impact on our assessment of the repeated switching of the ABL between the atria through the atrial septum during RFCA for AF.

TSP is an essential step in RFCA for AF and requires fluoroscopy guidance using LAO and RAO views [Earley 2009]. The ABL should be intentionally or unintentionally switched between the atria 2-4 times through the interatrial septum, particularly for the treatment of AF. Catheter slips from the left to the right atrium often occur when inexperienced operators perform ablation. Even in the hands of skilled operators, rapid arrhythmias, such as atrial tachycardia, atrial premature beats, and atrial flutter, are often induced during ablation of paroxysmal or persistent $\mathrm{AF}$ requiring excitation mapping and catheter movement between the left and right atria [Alhajiri 2014; Morady 2009]. Repeated atrial septum puncture increases the operation duration and $\mathrm{X}$-ray exposure duration; moreover, it greatly increases the risk of serious complications such as intraoperative cardiac tamponade [Holda 2016; KlimekPiotrowska 2016a; O'Brien 2017]. TSP is an important step during RFCA in patients with AF because improper TSP may cause erroneous pericardial tamponade or needle puncture of the patient's aorta, a life-threatening event. If TSP is performed repeatedly due to the requirement for mapping and ablation, the rate of complications may increase. The 
Table 3. Linear Ablation Data Obtained during Atrial Fibrillation Ablation

\begin{tabular}{|c|c|c|c|c|}
\hline LA roof ablation, $\mathrm{n}(\%)$ & $49(49.0)$ & $26(52.0)$ & $23(46)$ & .55 \\
\hline CTI linear ablation, $\mathrm{n}(\%)$ & $42(42)$ & $19(38.0)$ & $23(46.0)$ & .42 \\
\hline SVC ablation, n (\%) & $21(21.0)$ & $9(18.0)$ & $12(24.0)$ & .46 \\
\hline
\end{tabular}

LA indicates left atrial; MI, mitral isthmus; CTI, cavotricuspid isthmus; SVC, superior vena cava; CS, coronary sinus.

practical, 3D, show-catheter track-image function does not typically require repeated TSP and does not increase the risk of inappropriate puncture, thus reducing costs while not increasing the incidence of complications [Bigelow 2014]. It also obviates $\mathrm{X}$-ray dependence, thereby avoiding radiation damage to doctors and patients.

Yuan et al used fast anatomic mapping (FAM) via the Carto 3 system to reconstruct the LA and image-track between atria through a puncture site and achieved favorable results and ABL switching [Yuan 2017]. Their method and the current method are 3D systems that allow a fast path to be established between the left and right atria to facilitate ablation while reducing the dependence on $\mathrm{X}$-ray. FAM is more time-consuming and error-prone, while the image-track was displayed directly with the show-catheter function, and is a more precise method. A disadvantage of both methods is that if patients move their bodies, the $3 \mathrm{D}$ model is displaced, and the presented image-track will deviate from the actual position. With the use of ICE, fluoro-less TSP has come to be routinely used at some institutions. When it is necessary for the ABL to re-access the LA for mapping/ablation, the catheter can usually be readily advanced across the prior TSP site under ICE guidance. However, the use of ICE is not widespread due to its prohibitive cost.

\section{Study Limitations}

In this 1-year clinical study, we proved that ablation catheter switching between atria, guided by the image-track via the show-catheter function in the CARTO 3 system, is economical, safe, feasible, reproducible, and greatly reduces X-ray exposure. It is worthy of further investigation in multicenter studies with a larger patient population. The present study was limited in that it was a single-center study. Because different operators have different levels of proficiency, the operation time and $\mathrm{X}$-ray exposure duration for reintroducing the $\mathrm{ABL}$ into the LA will differ. Additionally, although different centers may use a point-by-point model or FAM for anatomical reconstruction of the LA, the show-catheter function is available and is suitable for FAM. For centers with access to ICE, this method may not be necessary.

\section{Conclusion}

We propose a practical method for ABL switching between atria, guided by the image-track via the show-catheter function in the CARTO 3 system during RF ablation of AF, either for mapping or ablation, which is economical, safe, feasible, and reproducible and greatly reduces $\mathrm{X}$-ray exposure time. This method is particularly suitable for centers that lack access to ICE.

\section{REFERENCES}

Alhajiri A, Ramadan MM, Senior R. 2014. Left atrial enlargement causing dysphagia and weight loss: a rare contraindication for catheter ablation therapy in a patient with complex atrial arrhythmia. Int J Cardiol 177:e111-12.

Bigelow AM, Smith G, Clark JM. 2014. Catheter ablation without fluoroscopy: Current techniques and future direction. J Atr Fibrillation 6:1066.

Chen S, Meng W, Sheng He D, et al. 2012. Blocking the pulmonary vein to left atrium conduction in addition to the entrance block enhances clinical efficacy in atrial fibrillation ablation. Pacing Clin Electrophysiol 35:524-31.

Chen S, Zhou G, Lu X, et al. 2019. The importance of identifying conduction breakthrough sites across the mitral isthmus by elaborate mapping for mitral isthmus linear ablation. Europace 21:950-60.

Christoph M, Wunderlich C, Moebius S, et al. 2015. Fluoroscopy integrated 3D mapping significantly reduces radiation exposure during ablation for a wide spectrum of cardiac arrhythmias. Europace 17:928-37.

Earley MJ. How to perform a transseptal puncture. 2009. Heart 95:85-92.

Gul EE, Baranchuk A, Glover BM. A guide to transseptal access. 2017. Can J Cardiol 33:544-7.

Hołda MK, Koziej M, Hołda J, et al. 2016. Anatomic characteristics of the mitral isthmus region: The left atrial appendage isthmus as a possible ablation target. Ann Anat 210:103-11.

Klimek-Piotrowska W, Hołda MK, Koziej M, et al. 2016. Clinical anatomy of the cavotricuspid isthmus and terminal crest. PLoS One 11(9):e0163383.

Klimek-Piotrowska W, Hołda MK, Koziej M, et al. 2016. Anatomy of the true interatrial septum for transseptal access to the left atrium. Ann Anat 205:60-4.

Meisinger QC, Stahl CM, Andre MP, Kinney TB, Newton IG. 2016. Radiation protection for the fluoroscopy operator and staff. AJR Am J Roentgenol 207:745-54.

Morady F, Oral H, Chugh A. 2009. Diagnosis and ablation of a typical 
atrial tachycardia and flutter complicating atrial fibrillation ablation. Heart Rhythm 6:S29-32.

O'Brien B, Zafar H, De Freitas S, Sharif F. 2017. Transseptal puncture

- Review of anatomy, techniques, complications and challenges. Int J
Cardiol 233:12-22.

Yuan Y, Long D, Sang C, Tao L, Dong J, Ma C. 2017. A practical guide for building a highway between atria during transseptal puncture without radiation. Anatol J Cardiol 17:470-3. 